\title{
68. MINERAL ANALYSES FROM THE PERIDOTITE-GABBRO-BASALT COMPLEX AT SITE 334, DSDP LEG 37
}

\author{
D. B. Clarke, Department of Geology, Dalhousie University, Halifax, Nova Scotia, Canada \\ and \\ H. Loubat, Département des Sciences de la Terre, Université de Quebec à Montréal, Montréal, P.Q.
}

\begin{abstract}
Fifty-one analyses of silicates, oxides, and sulfides from a suite of ultrabasic and basic rocks at Site 334 are presented. The intrusive rocks show rhythmic, cryptic, and phase layering and are closely analogous to the Rhum-layered intrusion. The extrusive rocks are mineralogically similar to the intrusives, but are unlikely to be genetically related to them.
\end{abstract}

\section{INTRODUCTION}

Drilling at Site 334 penetrated $\sim 50$ meters of basalts which overlie at least 68 meters of gabbros and peridotites. The basalts are generally aphyric, or sparsely porphyritic containing phenocrysts of olivine, clinopyroxene, and plagioclase. The presence of very coarse grained rocks at shallow depth under the basalts is in itself surprising and significant. The peridotites show well-developed cumulus textures in which olivine and spinel are always cumulus phases, whereas two pyroxenes and plagioclase are cumulus phases only in the gabbros. The present mineral analyses were undertaken to determine if the gabbros and peridotites are the complements of the overlying basalts, i.e., if there is a genetic relationship between the intrusive rocks below and the extrusive rocks above.

All analyses were done by electron microprobe (Cambridge Microscan MK-5) using natural minerals as standards. The raw data were processed using the EMPADR VII correction procceedure of Rucklidge and Gasparrini (1969).

\section{MINERAL ANALYSES}

Analyses of silicate minerals appear in Tables 1-3 and are graphically represented in Figure 1. In addition, Table 4 contains analyses of chrome spinels from the three peridotite specimens investigated. Also a qualitative petrographical investigation is summarized in Table 5. Finally, some of thee rocks were briefly studied for their sulfide mineralogy and the following two analyses were made in gabbro $334-21-1,47-49 \mathrm{~cm}$ :

\begin{tabular}{lcc} 
& Pyrite & Chalcopyrite \\
\cline { 2 - 3 } $\mathrm{Fe}$ & 47.83 & 30.63 \\
$\mathrm{Cu}$ & - & 34.54 \\
$\mathrm{Ni}$ & 0.07 & 0.03 \\
$\mathrm{~S}$ & $\underline{53.27}$ & $\underline{34.26}$ \\
\cline { 2 - 3 } & 101.18 & 99.47
\end{tabular}

In other rocks, the following sulfides were identified but not quantitatively analyzed: $22-2,61-63 \mathrm{~cm}$; pentlandite; $23-1,127-129 \mathrm{~cm}$; pyrrhotite; $24-3,112-114$ $\mathrm{cm}$; chalcopyrite, pyrite, pentlandite. In many cases the sulfides appear to occur as disseminated blebs enclosed within the primary silicate minerals.

\section{DISCUSSION}

Within the limitations of the visual core descriptions (Aumento and Melson, 1974) and the low degree of core recovery, there appear to be at least nine cycles of peridotite-olivine gabbro-gabbro in the plutonic complex of Site 334. A cycle usually begins with peridotite, but in some cases the most basic member may be olivine gabbro. Also, a cycle usually ends with olivinepoor or olivine-free gabbro, but in some cases the cycle ends while the gabbro is still relatively olivine rich. This kind of rhythmic layering is found in several layered basic intrusions from the terrestrial environment (Wager and Brown, 1968). It remains to be seen what the nature of the cryptic layering is and which terrestrial model most closely applies to the submarine sequence.

In Table 6 the samples are arranged in stratigraphic order and the mineral chemistry is presented in summary form. Samples 22-2, 61-63 cm and 21-1, 47-49 cm are from the same rhythmic unit, and it is apparent that there is normal cryptic variation from the peridotite to the gabbro, i.e., increasing Fs molecule in the pyroxenes. There is also elimination of olivine, presumably through a reaction relationship with the liquid at the 1 atmosphere invariant point, $\mathrm{O1}^{-}+\mathrm{Opx}^{+}+\mathrm{Cpx}^{+}+$ $\mathrm{Plag}^{+}+$Liquid $^{-}$. This reaction is strongly suggested by a careful petrographic examination: among the various episodes of crystallization, there is evidence of intensive replacement, suggested by frequent lobate contacts between olivine relics, pyroxenes, and plagioclase. It is also evident that whereas gabbro $21-1,47-49 \mathrm{~cm}$ is the uppermost gabbro in the sequence, it is not the most differentiated. The most evolved gabbro in terms of its Fs content in the pyroxenes is $23-1,127-129 \mathrm{~cm}$. A more primitive gabbro, $24-3,112-114 \mathrm{~cm}$, is found deeper in the sequence.

The reversal in the Fs content of the pyroxenes (and An content of the plagioclase) hints that the nine 
TABLE 1

Microprobe Analyses of Olivines

\begin{tabular}{|c|c|c|c|c|c|c|c|c|}
\hline & 1 & 2 & 3 & 4 & 5 & 6 & 7 & 8 \\
\hline Fo & 88.506 & 88.495 & 83.157 & 88.637 & 85.283 & 85.746 & 87.512 & 87.804 \\
\hline $\mathrm{Fa}$ & 11.254 & 11.279 & 16.446 & 10.884 & 14.295 & 13.989 & 12.382 & 12.116 \\
\hline Larn & 0.240 & 0.227 & 0.397 & 0.479 & 0.422 & 0.265 & 0.106 & 0.080 \\
\hline Fo & 88.719 & 88.696 & 83.489 & 89.063 & 85.644 & 85.463 & 87.274 & 87.551 \\
\hline $\mathrm{Fa}$ & 11.281 & 11.304 & 16.511 & 10.937 & 14.356 & 13.943 & 12.348 & 12.081 \\
\hline Niol & 0.000 & 0.000 & 0.000 & 0.000 & 0.000 & 0.594 & 0.378 & 0.368 \\
\hline $\mathrm{F} / \mathrm{M}$ & 0.127 & 0.127 & 0.198 & 0.123 & 0.168 & 0.163 & 0.141 & 0.138 \\
\hline $\mathrm{F} / \mathrm{FM}$ & 0.113 & 0.113 & 0.165 & 0.109 & 0.144 & 0.140 & 0.124 & 0.121 \\
\hline
\end{tabular}

Note: $1=26-1,20-22 \mathrm{~cm}$; olivine $5.2=26-1,20-22 \mathrm{~cm}$; olivine $6.3=15-2,20 \mathrm{~cm}$; olivine $1.4=15-2,20 \mathrm{~cm} ;$ olivine $2.5=20-2,140-145 \mathrm{~cm} ;$ olivine 1. $6=24-3,112-114 \mathrm{~cm}$; olivine $1.7=22-2,61-63 \mathrm{~cm}$; olivine $1.8=22-2,61-63 \mathrm{~cm}$ : olivine 2 .

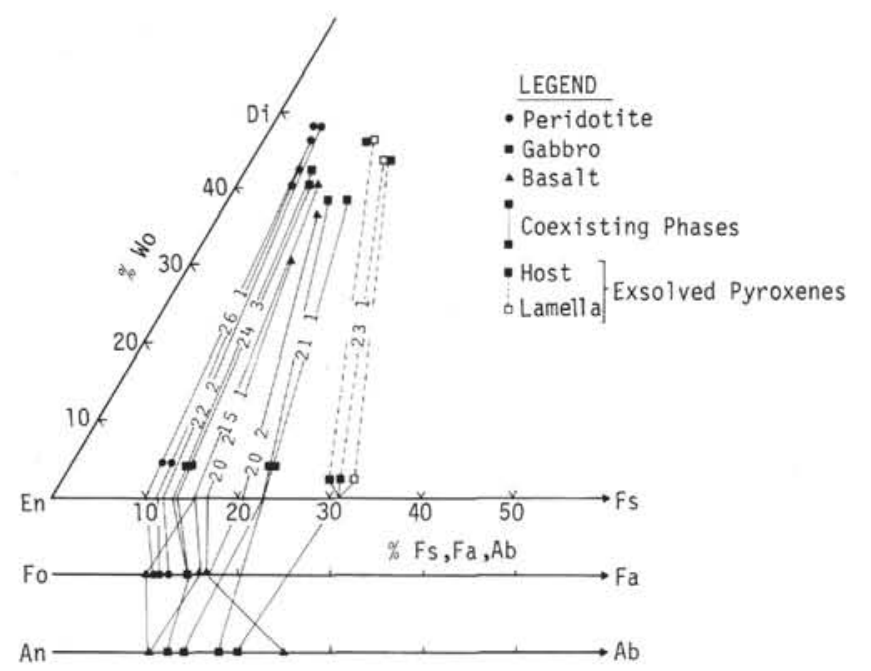

Figure 1. Mineral analyses for intrusive and extrusive rocks at Site 334.

rhythmic units are not the result of fractional crystallization of a single magma as is the case in the Skaergaard intrusion. Instead, the cyclical variation of rock types and mineral chemistry suggests that this complex has formed in a manner similar to that described for the Rhum-layered intrusion by Brown (1956). It is believed that these cumulates formed in a magma chamber that acted as a temporary reservoir for magmas en route to the surface. The longer the magma stayed in the reservoir, the more evolved would become both the cumulate pile and the erupted basalt.

There is no compelling mineralogical evidence to prove or disprove a genetic link between the basalts and the underlying intrusives. The basalts are more erratic in their mineral chemistry principally because most phenocrysts in the basalts suggest that, if there is a genetic link, these basalts were erupted from the magma chamber at a stage of evolution between gabbros of the types $24-3,112-114 \mathrm{~cm}$ and $21-1,47-49 \mathrm{~cm}$. However, more convincing evidence exists against the idea that there is any genetic relationship between the intrusives and extrusives at Site 334. First, the close spatial relationship between the two groups makes unlikely the notion that the coarse-grained intrusives could be in situ cumulates from the same magmas that gave rise to the basalts. Secondly, the numerous breccia zones noted in the core logs, plus the cataclastic tex- tures in the gabbros, suggest that these cumulates were tectonically emplaced. The greater the degree of displacement this type of deformation represents, the less likely it is that the intrusives and extrusives are genetically related. Among the breccias, the mysterious nannofossil chalk matrix could tentatively be explained by a process of "oblique diapirism" of ultramafic lenses being serpentinized. The degree of serpentinization of olivine is a direct function of the amount of olivine in these rocks (i.e., a dunitic layer is totally serpentinized, and an olivine-poor gabbro has fresh olivine). If serpentinization occurred with an increase of volume, it could result in a dislocation of the layered sequence, across an oblique serpentinization front (Loubat, 1973). Lenses of brecciated serpentinites would eventually reach very superficial levels of the oceanic floor (Figure 2).

\section{CONCLUSIONS}

The conclusions listed below are tentative because of the gaps in the core record and because of the limited mineral data.

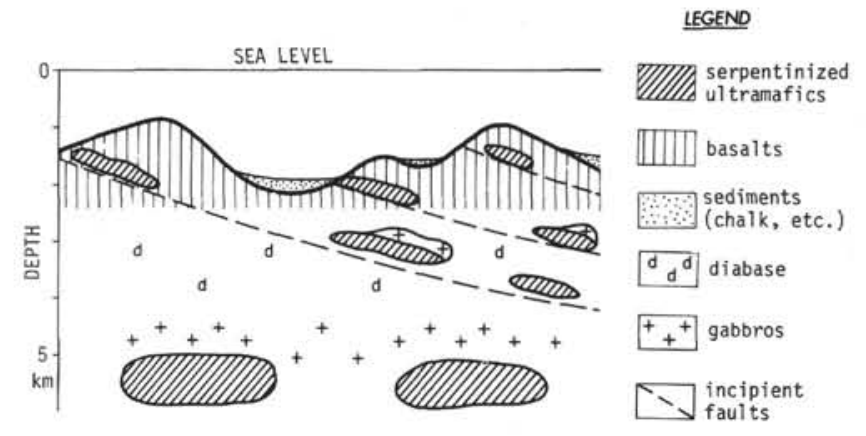

Figure 2. Proposed shallow cross-section of the oceanic floor at some distance away from the axis of a volcanic ridge. Due to steeply oblique isotherms and serpentinization fronts, a lateral increase of volume and dislocation of ultramafic layers could occur. Some of these bodies in the process of serpentinization will eventually be dragged in a phenomenon of "oblique diapirism," and they could reach levels of superficial basalts and sediments, in a process of incipient dislocation of the oceanic floor. This could account for the differential degree of serpentinization of the layered body, the obvious increase of volume in the serpentinization, and the breccias with chalky fossiliferous matrix. 
TABLE 2

Microprobe Analyses of Pyroxenes

\begin{tabular}{|c|c|c|c|c|c|c|c|c|c|c|c|c|c|c|c|c|}
\hline & \multicolumn{2}{|c|}{1} & \multicolumn{2}{|c|}{2} & \multicolumn{2}{|c|}{3} & \multicolumn{2}{|c|}{4} & \multicolumn{2}{|c|}{5} & \multicolumn{2}{|c|}{6} & \multicolumn{2}{|c|}{7} & \multicolumn{2}{|c|}{8} \\
\hline $\mathrm{SiO}_{2}$ & \multicolumn{2}{|c|}{53.69} & \multicolumn{2}{|c|}{53.32} & \multicolumn{2}{|c|}{54.37} & 52.3 & & 52. & & 42 & & & & & \\
\hline $\mathrm{TiO}_{2}$ & & 20 & & & & 20 & 0.4 & & 0 . & & & 41 & & 21 & & 37 \\
\hline $\mathrm{Al}_{2} \mathrm{O}_{3}$ & & 19 & & 75 & & 70 & 1.5 & & 2. & & & 84 & & 14 & & 97 \\
\hline $\mathrm{Cr}_{2} \mathrm{O}_{3}$ & & 07 & & & & 05 & 0.0 & & 0 . & & & 08 & & 06 & & 33 \\
\hline $\mathrm{Fe}_{2} \mathrm{O}_{3}$ & & .00 & & & & 00 & 0.0 & & 0. & & & 00 & & 00 & & 00 \\
\hline $\mathrm{FeO}$ & & 85 & & & & & 8.5 & & 7. & & & 54 & & & & 72 \\
\hline $\mathrm{MnO}$ & & 23 & & & & 24 & 0.2 & & 0 . & & & 23 & & 29 & & 00 \\
\hline $\mathrm{MgO}$ & & 09 & & & & & 14.9 & 7 & 14. & & & & & 35 & & \\
\hline $\mathrm{CaO}$ & & 65 & & & & 72 & 21.5 & & 21 . & & & & & 10 & & \\
\hline $\mathrm{Na}_{2} \mathrm{O}$ & & 07 & & & & 08 & 0.2 & & 0 . & & & 26 & & 09 & & 90 \\
\hline $\mathrm{K}_{2} \mathrm{O}$ & & 02 & & 04 & & 03 & 0.0 & & 0 . & & & 06 & & 03 & & 03 \\
\hline Sum & & 98 & 100 & & 100 & & 99.9 & & 99. & & & & 100 & & 100 & \\
\hline $\mathrm{Si}$ & 1.966 & * & 1.961 & * & 1.985 & * & 1.950 & * & 1.953 & * & 1.952 & * & 1.973 & * & 1.940 & * \\
\hline $\mathrm{Al}$ & 0.034 & 2.000 & 0.039 & 2.000 & 0.014 & 2.000 & 0.050 & 2.000 & 0.047 & 2.000 & 0.048 & 2.000 & 0.027 & 2.000 & 0.060 & 2.000 \\
\hline $\mathrm{Al}$ & 0.014 & $*$ & 0.037 & $*$ & 0.016 & $*$ & 0.018 & * & 0.051 & * & 0.033 & $*$ & 0.022 & * & 0.067 & $*$ \\
\hline $\mathrm{Ti}$ & 0.006 & * & 0.009 & * & 0.005 & * & 0.011 & * & 0.008 & * & 0.012 & * & 0.006 & * & 0.000 & * \\
\hline $\mathrm{Cr}$ & 0.002 & * & 0.003 & * & 0.001 & $*$ & 0.003 & $*$ & 0.004 & * & 0.002 & * & 0.002 & * & 0.038 & * \\
\hline $\mathrm{Fe} 3+$ & 0.000 & $*$ & 0.000 & * & 0.000 & * & 0.000 & * & 0.000 & * & 0.000 & $*$ & 0.000 & * & 0.000 & * \\
\hline $\mathrm{Fe}$ & 0.578 & * & 0.235 & * & 0.597 & * & 0.265 & * & 0.220 & * & 0.266 & $*$ & 0.626 & * & 0.082 & * \\
\hline $\mathrm{Mn}$ & 0.007 & * & 0.008 & * & 0.007 & * & 0.007 & * & 0.006 & * & 0.007 & * & 0.009 & * & 0.000 & * \\
\hline $\mathrm{Mg}$ & 1.369 & 1.975 & 0.814 & 1.105 & 1.334 & 1.961 & 0.831 & 1.134 & 0.827 & 1.116 & 0.800 & 1.120 & 1.284 & 1.949 & 0.884 & 1.081 \\
\hline $\mathrm{Ca}$ & 0.026 & $*$ & 0.876 & $=$ & 0.028 & * & 0.859 & * & 0.861 & * & 0.864 & * & 0.043 & * & 0.854 & $*$ \\
\hline $\mathrm{Na}$ & 0.005 & * & 0.019 & $*$ & 0.006 & * & 0.019 & * & 0.019 & * & 0.019 & * & 0.006 & * & 0.063 & * \\
\hline K & 0.001 & 0.031 & 0.002 & 0.895 & 0.001 & 0.035 & 0.002 & 0.880 & 0.002 & 0.883 & 0.003 & 0.886 & 0.001 & 0.051 & 0.001 & 0.918 \\
\hline o & 6.000 & $*$ & 6.000 & $*$ & 6.000 & * & 6.000 & $*$ & 6.000 & $*$ & 6.000 & * & 6.000 & * & 6.000 & $*$ \\
\hline wo & & 293 & & 512 & & 439 & 43.9 & & 45. & & & 771 & & 226 & & 917 \\
\hline EN & & 426 & & 295 & & 102 & 42.5 & & 43. & & & 426 & & 729 & & 551 \\
\hline FS & & 281 & & 193 & & 459 & 13.5 & & 11. & & & 804 & & 046 & & 532 \\
\hline wo & & 285 & & 398 & & 429 & 43.3 & & 44. & & & 174 & & 208 & & 341 \\
\hline HYP & & 465 & & 152 & & 284 & 55.6 & & 54. & & & 865 & & 465 & & 293 \\
\hline JD & & 250 & & 950 & & 287 & 0.9 & & & & & 961 & & 327 & & 360 \\
\hline $\mathrm{F} / \mathrm{M}$ & & 427 & & 298 & & 453 & 0.3 & & & & & 431 & & 495 & & 93 \\
\hline F/FM & & 299 & & 229 & & 312 & 0.2 & 46 & & & & 255 & & 331 & & 85 \\
\hline & & 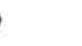 & & & & & 12 & & 1 & & 1 & & & & & \\
\hline $\mathrm{SiO}_{2}$ & & 73 & & & & & 51.2 & & 50. & & & & & & & \\
\hline $\mathrm{TiO}_{2}$ & & 11 & & & & 22 & 0.2 & & 0 . & & & 25 & & 22 & & 21 \\
\hline $\mathrm{Al}_{2} \mathrm{O}_{3}$ & & 14 & & & & 69 & 3.1 & & 2. & & & 24 & & 12 & & 99 \\
\hline $\mathrm{Cr}_{2} \mathrm{O}_{3}$ & & 77 & & & & 27 & 1.2 & & 0 . & & & 24 & & 09 & & 10 \\
\hline $\mathrm{Fe}_{2} \mathrm{O}_{3}$ & & 00 & & & & 00 & 0.0 & & 0. & & & 00 & & 00 & & 00 \\
\hline $\mathrm{FeO}$ & & 79 & & & & 64 & 3.3 & & 6. & & & 96 & & & & \\
\hline $\mathrm{MnO}$ & & 00 & & & & 11 & 0.1 & & 0 . & & & 20 & & 11 & & 11 \\
\hline $\mathrm{MgO}$ & & 48 & & & & & 17.9 & & 17. & & & & & 88 & & \\
\hline $\mathrm{CaO}$ & & 53 & & & & & 20.7 & & 18. & & & & & 03 & & 98 \\
\hline $\mathrm{Na}_{2} \mathrm{O}$ & & 03 & & & & 15 & 0.2 & & 0. & & & 14 & & 01 & & 1 \\
\hline $\mathrm{K}_{2} \mathrm{O}$ & & 01 & & & & 06 & 0.0 & & 0 . & & & 05 & & 03 & & 02 \\
\hline Sum & & 59 & 100 & & & & 98.3 & & 97. & & & & & 22 & & \\
\hline $\mathrm{Si}$ & 1.948 & $*$ & 1.944 & $*$ & 1.880 & * & 1.896 & * & 1.904 & * & 1.926 & * & 1.950 & * & 1.947 & * \\
\hline $\mathrm{Al}$ & 0.052 & 2.000 & 0.056 & 2.000 & 0.120 & 2.000 & 0.104 & 2.000 & 0.096 & 2.000 & 0.074 & 2.000 & 0.049 & 1.999 & 0.048 & 1.995 \\
\hline Al & 0.036 & $*$ & 0.032 & $*$ & 0.042 & * & 0.034 & $*$ & 0.023 & $*$ & 0.027 & $*$ & 0.000 & * & 0.000 & * \\
\hline $\mathrm{Ti}$ & 0.003 & * & 0.003 & $*$ & 0.006 & * & 0.007 & * & 0.005 & $*$ & 0.007 & $*$ & 0.006 & * & 0.006 & * \\
\hline $\mathrm{Cr}$ & 0.021 & * & 0.020 & * & 0.037 & * & 0.037 & * & 0.012 & * & 0.007 & $*$ & 0.003 & * & 0.003 & $*$ \\
\hline $\mathrm{Fe} 3+$ & 0.000 & $*$ & 0.000 & $*$ & 0.000 & * & 0.000 & * & 0.000 & * & 0.000 & $*$ & 0.000 & * & 0.000 & * \\
\hline $\mathrm{Fe}$ & 0.198 & $*$ & 0.194 & $*$ & 0.113 & $*$ & 0.014 & * & 0.217 & * & 0.253 & * & 0.439 & * & 0.440 & * \\
\hline Mn & 0.000 & $*$ & 0.000 & $*$ & 0.003 & * & 0.003 & * & 0.005 & $*$ & 0.006 & * & 0.003 & $*$ & 0.003 & $*$ \\
\hline $\mathrm{Mg}$ & 1.640 & 1.899 & 1.666 & 1.915 & 1.027 & 1.228 & 0.993 & 1.178 & 1.002 & 1.263 & 0.962 & 1.262 & 1.486 & 1.937 & 1.495 & 1.947 \\
\hline $\mathrm{Ca}$ & 0.095 & $*$ & 0.082 & * & 0.779 & * & 0.823 & $*$ & 0.756 & $*$ & 0.744 & $*$ & 0.081 & $*$ & 0.079 & * \\
\hline $\mathrm{Na}$ & 0.002 & $*$ & 0.003 & * & 0.011 & * & 0.014 & $*$ & 0.010 & $*$ & 0.010 & $*$ & 0.001 & * & 0.001 & $*$ \\
\hline K & 0.000 & 0.097 & 0.001 & 0.086 & 0.003 & 0.793 & 0.003 & 0.841 & 0.002 & 0.769 & 0.002 & 0.757 & 0.001 & 0.083 & 0.001 & 0.030 \\
\hline 0 & 6.000 & * & 6.000 & * & 6.000 & * & 6.000 & * & 6.000 & * & 6.000 & $*$ & 6.000 & * & 6.000 & $*$ \\
\hline
\end{tabular}

1) The sequence of nine rhythmic units in the intrusive rocks at Site 334 appears to be the product of a Rhum, rather than a Skaergaard, type of fractional crystallization.

2) Cryptic variation is present in the uppermost intrusive unit, and it probably exists in each of the rhythmic units. A detailed study of the phase chemistry of each rhythmic unit is suggested in order that the full record of evolution may be understood.

3) There is phase layering in many of the units; olivine and chrome spinel are frequently observed at the bottom, but not the top, of individual rhythmic 
TABLE 2 - Continued

\begin{tabular}{|c|c|c|c|c|c|c|c|c|c|c|c|c|c|c|c|c|c|c|}
\hline & \multicolumn{2}{|c|}{9} & \multicolumn{2}{|c|}{10} & \multicolumn{2}{|c|}{11} & \multicolumn{2}{|c|}{12} & \multicolumn{2}{|c|}{13} & \multicolumn{2}{|c|}{14} & \multicolumn{2}{|c|}{15} & \multicolumn{2}{|c|}{16} & & \\
\hline wo & \multicolumn{2}{|c|}{4.901} & \multicolumn{2}{|c|}{4.200} & \multicolumn{2}{|c|}{40.510} & \multicolumn{2}{|c|}{42.872} & \multicolumn{2}{|c|}{38.293} & \multicolumn{2}{|c|}{37.979} & \multicolumn{2}{|c|}{4.023} & \multicolumn{2}{|c|}{3.911} & & \\
\hline EN & \multicolumn{2}{|c|}{84.833} & \multicolumn{2}{|c|}{85.784} & 53 & 491 & 51.7 & & 50 . & & 49 & 105 & 74 & 105 & & & & \\
\hline FS & 10. & 266 & & & & 899 & 5.4 & & 10. & & 12 & 916 & 21 & 872 & & & & \\
\hline wo & & 896 & & 193 & 40. & 310 & 42.4 & & 37. & & 37 & 657 & & .015 & & 03 & & \\
\hline HYP & 94 & 999 & & & 59 & 131 & 56.7 & & 61. & & 61 & 821 & 95 & .950 & & 61 & & \\
\hline JO & & 105 & & 173 & & 559 & 0.7 & & & & & 522 & & .036 & & 36 & & \\
\hline $\mathrm{F} / \mathrm{M}$ & & 121 & & 117 & & 114 & 0.1 & & & & & 270 & & 297 & & 95 & & \\
\hline $\mathrm{F} / \mathrm{FM}$ & & 108 & & 105 & & 102 & 0.0 & & & & & 212 & & 229 & & 29 & & \\
\hline & 1 & & 1 & & 1 & & 20 & & 2 & & 2 & & 2 & & 2 & & 2 & \\
\hline $\mathrm{SiO}_{2}$ & 52. & & 51 & & 52 & & 54.9 & & 55 & & 52 & & 52 & 90 & 52 & & 51. & \\
\hline $\mathrm{TiO}_{2}$ & & 43 & & & 0 . & 00 & 0.3 & & 0 . & & & 40 & & 41 & & & & \\
\hline $\mathrm{Al}_{2} \mathrm{O}_{3}$ & & 70 & & & & 81 & 1.6 & & 1. & & & 68 & & 55 & & & & \\
\hline $\mathrm{Cr}_{2} \mathrm{O}_{3}$ & & 00 & & & & 00 & 0.4 & & 0 . & & & 66 & & 57 & & & & \\
\hline $\mathrm{Fe}_{2} \mathrm{O}_{3}$ & & 00 & & & & 00 & 0.0 & & 0. & & & 00 & & .00 & & & & \\
\hline $\mathrm{FeO}$ & & 80 & & & & 60 & 8.4 & & 8 & & & 91 & & .29 & & & & \\
\hline $\mathrm{MnO}$ & & 15 & & & & 00 & 0.3 & & 0. & & & 34 & & 34 & & & & \\
\hline $\mathrm{MgO}$ & 17 & & 18 & & 20 & 88 & 31. & & 30. & & 18 & & 17 & 35 & 16 & & 16. & \\
\hline $\mathrm{CaO}$ & 19 & & 17 & & 14 & .71 & 2.4 & & 2 & & 19 & & 20 & .27 & 23 & & 23. & \\
\hline $\mathrm{Na}_{2} \mathrm{O}$ & & 18 & & & & 13 & 0.1 & & 0 & & & 27 & & .27 & & & & \\
\hline $\mathrm{K}_{2} \mathrm{O}$ & & 06 & & & & 00 & 0.0 & & 0 . & & & 09 & & .09 & & & & \\
\hline Sum & 100 & & 99 & & 98 & 27 & 99.9 & & 99. & & 100 & & 99 & .04 & 99 & & 99. & \\
\hline $\mathrm{Si}$ & 1.902 & $*$ & 1.868 & $*$ & 1.913 & * & 1.934 & * & 1.942 & * & 1.913 & * & 1.943 & * & 1.925 & * & 1.909 & * \\
\hline Al & 0.098 & 2.000 & 0.132 & 2.000 & 0.087 & 2.000 & 0.066 & 2.000 & 0.058 & 2.000 & 0.087 & 2.000 & 0.057 & 2.000 & 0.074 & 2.000 & 0.091 & 2.000 \\
\hline Al & 0.060 & * & 0.108 & $*$ & 0.078 & * & 0.004 & $*$ & 0.016 & • & 0.028 & $*$ & 0.053 & * & 0.039 & $*$ & 0.050 & * \\
\hline $\mathrm{Ti}$ & 0.012 & • & 0.000 & $*$ & 0.000 & $*$ & 0.009 & * & 0.019 & * & 0.011 & $*$ & 0.011 & * & 0.008 & * & 0.009 & * \\
\hline $\mathrm{Cr}_{\mathrm{r}}$ & 0.000 & $*$ & 0.000 & * & 0.000 & * & 0.011 & * & 0.011 & * & 0.019 & $*$ & 0.017 & * & 0.024 & * & 0.021 & $*$ \\
\hline $\mathrm{Fe} 3+$ & 0.000 & * & 0.000 & * & 0.000 & * & 0.000 & * & 0.000 & * & 0.000 & * & 0.010 & * & 0.000 & * & 0.000 & * \\
\hline $\mathrm{Fe}$ & 0.177 & $*$ & 0.198 & * & 0.202 & * & 0.249 & * & 0.245 & * & 0.149 & $*$ & 0.132 & * & 0.156 & * & 0.097 & * \\
\hline $\mathrm{Mn}$ & 0.005 & * & 0.000 & * & 0.000 & * & 0.011 & $\cdot$ & 0.011 & * & 0.010 & * & 0.011 & - & 0.004 & * & 0.007 & * \\
\hline $\mathrm{Mg}$ & 0.970 & 1.223 & 1.002 & 1.308 & 1.142 & 1.422 & 1.634 & 1.919 & 1.615 & 1.907 & 1.015 & 1.233 & 0.960 & 1.173 & 0.891 & 1.072 & 0.888 & 1.072 \\
\hline $\mathrm{Ca}$ & 0.776 & * & 0.699 & * & 0.578 & $*$ & 0.091 & * & 0.093 & $*$ & 0.765 & $*$ & 2.798 & $*$ & 0.917 & * & 0.920 & * \\
\hline $\mathrm{Na}$ & 0.013 & * & 0.010 & * & 0.009 & * & 0.010 & * & 0.010 & * & 0.019 & $*$ & 0.019 & $\bullet$ & 0.014 & $*$ & 0.015 & * \\
\hline $\mathrm{K}$ & 0.003 & 0.792 & 0.000 & 0.709 & 0.000 & 0.587 & 0.004 & 0.104 & 0.004 & 0.107 & 0.004 & 0.788 & 0.004 & 0.321 & 0.003 & 0.934 & 0.003 & 0.938 \\
\hline 0 & 6.000 & $*$ & 6.000 & * & 6.900 & $*$ & 6.000 & * & 6.000 & $*$ & 6.000 & $*$ & 6.000 & $*$ & 6.000 & $*$ & 6.000 & * \\
\hline wo & 40 & 364 & 36 & 800 & 30 & 076 & 4.6 & & & 48 & 39 & 653 & 42. & .446 & & 26 & 48. & 283 \\
\hline EN & 50 & 453 & & 772 & 59. & 391 & 82.7 & & 82 & & 52 & 606 & 50. & .542 & & 548 & 46. & 501 \\
\hline FS & & 183 & & 428 & 10 & 533 & 12.6 & & 12. & & & 742 & & .012 & & 25 & & 116 \\
\hline wo & 40. & 004 & & 609 & 29 & 932 & 4.5 & & & 97 & 39 & 055 & 41 & .783 & & 80 & 47. & 728 \\
\hline HYP & 59 & 342 & & 871 & 69 & 589 & 94.9 & & 94 & & 59 & 973 & 57 & .210 & & 32 & 51. & 495 \\
\hline JD & & 655 & & 520 & & 479 & 0.4 & & & 18 & & 972 & & .007 & & 733 & & 777 \\
\hline $\mathrm{F} / \mathrm{M}$ & & 187 & & 198 & & 177 & 0.1 & & & 59 & & 157 & & 150 & & 123 & & 118 \\
\hline $\mathrm{F} / \mathrm{FM}$ & & 157 & & 165 & & 151 & 0.1 & & & 37 & & 136 & & 130 & & 109 & & 105 \\
\hline
\end{tabular}

Note: $1=23-1,127-129 \mathrm{~cm}$; OPX host $1.2=23-1,127-129 \mathrm{~cm} ;$ CPX lamella $1 \mathrm{~A} .3=23-1,127-129 \mathrm{~cm} ;$ OPX host $2.4=23-1,127-129 \mathrm{~cm} ; \mathrm{CPX}$ lamella $2 \mathrm{~A} .5=23-1$, $127-129 \mathrm{~cm}$; CPX host $3.6=23-1,127-129 \mathrm{~cm} ;$ CPX host $4.7=23-1,127-129 \mathrm{~cm} ;$ OPX lamella $4 \mathrm{~A}, 8=26-1,20-22 \mathrm{~cm} ; \mathrm{OPX} 3.9=26-1,20-22 \mathrm{~cm} ; \mathrm{OPX} 7.10=26$ $1,20-22 \mathrm{~cm} ;$ OPX $8.11=26-1,20-22 \mathrm{~cm} ;$ CPX $9.12=26-1,20-22 \mathrm{~cm} ;$ CPX $10.13=21-1,47-49 \mathrm{~cm} ;$ CPX $1.14=21-1,47-49 \mathrm{~cm} ;$ CPX $2.15=21-1,47-49 \mathrm{~cm} ;$ OPX 3. $16=21-1,47-49 \mathrm{~cm} ;$ OPX $4.17=15-2,30 \mathrm{~cm} ; \mathrm{CPX} 1.18=20-2,140-145 \mathrm{~cm} ; \mathrm{CPX} 3.19=20-2,140-145 \mathrm{~cm} ; \mathrm{CPX} 4.20=24-3,112-144 \mathrm{~cm} ; \mathrm{OPX} 2.21=24-3,112-$ $114 \mathrm{~cm} ;$ OPX $3.22=24-3,112-114 \mathrm{~cm} ; \operatorname{CPX} 4.23=24-3,112-114 \mathrm{~cm} ; \mathrm{CPX} 5.24=22-2,61-63 \mathrm{~cm} ; \mathrm{CPX} 3.25=22-2,61-63 \mathrm{~cm} ; \mathrm{CPX} 4$.

units. This phase layering is attributed to reaction relationships in a fractionating magma rather than settling of dense crystals in rhythmic units developed by "turbidity currents" in the magma chamber.

4) The mineral chemistry alone is not sufficient to establish or disprove a genetic link between the basalts and the underlying intrusives.

5) A genetic link between intrusives and extrusives is unlikely because of the close spatial association of the rocks and because of the deformation of the intrusives suggesting that they have been tectonically emplaced.

\section{REFERENCES}

Aumento, F. and Melson, W.G., 1974. DSDP Leg 37 visual core descriptions and sample distribution for igneous cores, Holes 332A, 332B, 333A, 334, 335: DSDP Leg 37 Shipboard Report.

Brown, G.M., 1956. The layered ultrabasic rocks of Rhum, inner Hebrides: Phil. Trans. Roy. Soc. London, Series 240B, p. $1-53$.

Loubat, H., 1973. Soubassement des dorsales volcaniques océaniques: Suisse Min. Pétrol. Bull., v. 53, p. 337-354.

Rucklidge, J. and Gasparrini, E.L., 1969. Electron microprobe analytical data reduction: Dept. of Geology, Institute of Toronto, Canada.

Wager, L.R. and Brown, G.M., 1968. Layered igneous rocks: Edinburgh (Oliver and Boyd). 
TABLE 3

Microprobe Analyses of Feldspars

\begin{tabular}{|c|c|c|c|c|c|c|c|c|c|c|c|c|c|c|c|c|c|c|c|c|}
\hline & \multicolumn{2}{|c|}{1} & \multicolumn{2}{|c|}{2} & \multicolumn{2}{|c|}{3} & \multicolumn{2}{|c|}{4} & \multicolumn{2}{|c|}{5} & \multicolumn{2}{|c|}{6} & \multicolumn{2}{|c|}{7} & \multicolumn{2}{|c|}{8} & \multicolumn{2}{|c|}{9} & \multicolumn{2}{|c|}{10} \\
\hline $\mathrm{SiO}_{2}$ & \multicolumn{2}{|c|}{48.19} & \multicolumn{2}{|c|}{47.30} & \multicolumn{2}{|c|}{47.57} & \multicolumn{2}{|c|}{47.00} & \multicolumn{2}{|c|}{46.87} & \multicolumn{2}{|c|}{48.30} & \multicolumn{2}{|c|}{46.47} & \multicolumn{2}{|c|}{51.33} & \multicolumn{2}{|c|}{50.41} & \multicolumn{2}{|c|}{45.72} \\
\hline $\mathrm{TiO}_{2}$ & \multicolumn{2}{|c|}{0.05} & \multicolumn{2}{|c|}{0.05} & \multicolumn{2}{|c|}{0.04} & \multicolumn{2}{|c|}{0.04} & 0. & & 0.8 & & & 00 & 0. & & 0.0 & & & 02 \\
\hline $\mathrm{Al}_{2} \mathrm{O}_{3}$ & 32. & & 32. & & 32. & & 32. & & 32. & & 32.1 & & 33 & & 29. & & 29.9 & & 33. & \\
\hline $\mathrm{Cr}_{2} \mathrm{O}_{3}$ & 0. & & 0. & & 0. & & 0. & 00 & 0. & & $0 . c$ & & & 00 & 0. & & 0.0 & & & 00 \\
\hline $\mathrm{Fe}_{2} \mathrm{O}_{3}$ & 0. & & 0. & & 0.0 & & 0. & 00 & 0. & & 0. & & & 00 & 0. & & 0.0 & & & 00 \\
\hline $\mathrm{FeO}$ & 0.0 & & 0. & & 0. & & 0. & 00 & 0. & 0 & $0 . c$ & & & 29 & 0. & & 0.7 & & & 00 \\
\hline $\mathrm{MnO}$ & 0. & & 0. & & 0. & & 0. & 00 & 0. & & 0.0 & & & 00 & 0. & & 0.0 & & & 00 \\
\hline $\mathrm{MgO}$ & & 00 & 0. & & 0. & & 0. & 00 & 0. & & $0 .($ & & & 33 & 0. & & 0.5 & & & 00 \\
\hline $\mathrm{CaO}$ & 16. & & 15. & & 16.5 & & 17. & & 17. & & 16.5 & & 18 & & 14.8 & & 15.4 & & 18. & \\
\hline $\mathrm{Na}_{2} \mathrm{O}$ & 2. & & 2. & & 1.5 & & 1. & 66 & 1. & & 2.1 & & & 12 & 2. & & 2.6 & & & 32 \\
\hline $\mathrm{K}_{2} \mathrm{O}$ & 0. & & 0. & & $0 . c$ & & 0. & 06 & 0. & & 0.0 & & & 00 & 0. & & 0.0 & & & 05 \\
\hline Sum & 99.2 & & 99. & & 99.2 & & 99. & & 98. & & 99.2 & & 100 & & 99. & & 99.6 & & 98. & \\
\hline $\mathrm{Si}$ & 8.900 & * & 8.837 & * & 8.795 & * & 8.787 & * & 8.711 & * & 8.916 & * & 8.553 & * & 9.409 & * & 9.259 & * & 8.541 & * \\
\hline $\mathrm{Ti}$ & 0.887 & * & 0.317 & * & 0.006 & * & 0.006 & $*$ & 0.007 & * & 0.007 & * & 0.000 & * & 0.000 & * & 0.000 & $*$ & 0.003 & * \\
\hline $\mathrm{Al}$ & 6.992 & * & 7.072 & * & 7.113 & * & 7.188 & * & 7.168 & * & 6.985 & * & 7.304 & * & 6.348 & * & 6.488 & $*$ & 7.391 & * \\
\hline Fe3+ & 0.000 & * & 0.000 & * & 0.000 & * & 0.000 & * & 0.000 & * & 0.000 & * & 0.000 & * & 0.000 & * & 0.000 & * & 0.000 & * \\
\hline $\mathrm{Fe}$ & 0.000 & * & 0.000 & * & 0.000 & * & 0.000 & * & 0.000 & * & 0.000 & * & 0.045 & * & 0.117 & * & 0.114 & * & 0.000 & * \\
\hline Mn & 0.000 & * & 0.000 & * & 0.000 & * & 0.000 & * & 0.000 & * & 0.000 & * & 0.000 & $*$ & 0.000 & * & 0.000 & * & 0.000 & * \\
\hline $\mathrm{Mg}$ & 0.000 & 15.899 & 0.000 & 15.916 & 0.000 & 15.914 & 0.000 & 15.901 & 0.000 & 15.866 & 0.000 & 15.908 & 0.091 & 15.992 & 0.158 & 16.033 & 0.140 & 16.000 & 0.000 & 15.895 \\
\hline $\mathrm{Ca}$ & 3.287 & * & 3.308 & * & 3.364 & * & 3.483 & * & 3.603 & * & 3.281 & * & 3.599 & $*$ & 2.917 & * & 3.030 & * & 3.637 & * \\
\hline $\mathrm{Na}$ & 0.798 & * & 0.771 & * & 0.786 & * & 0.596 & * & 0.598 & * & 0.766 & * & 0.400 & * & 0.928 & * & 0.926 & * & 0.478 & * \\
\hline K & 0.016 & 4.102 & 0.014 & 4.093 & 0.017 & 4.086 & 0.014 & 4.094 & 0.014 & 4.115 & 0.016 & 4.064 & 0.000 & 3.998 & 0.000 & 3.844 & 0.000 & 3.956 & 0.312 & 4.127 \\
\hline $\mathrm{O}$ & 32.000 & * & 32.000 & * & 32.000 & * & 32.000 & * & 32.000 & * & 32.000 & * & 32.000 & * & 32.000 & * & 32.000 & * & 32.000 & * \\
\hline Or & & 02 & & & 0.4 & 84 & & 346 & & 346 & 0.4 & 06 & & 000 & & 00 & 0.0 & 00 & & 283 \\
\hline $\mathrm{AB}$ & 19. & & 18. & & 17.2 & & 14. & 564 & 14. & & 18.8 & & & 995 & 24.1 & & 23.4 & & 11. & 585 \\
\hline AN & 80. & & 80. & & 82. & & 84. & 990 & 85. & & 80.7 & & & 005 & 75.8 & & 76.5 & & 88. & 126 \\
\hline $\mathrm{QZ}$ & 91. & & 91. & & 92. & & 93. & 449 & 93. & & 91.5 & & & 536 & 91.0 & & 90.9 & & 94. & 574 \\
\hline NE & 8. & 219 & & 100 & 7.4 & 20 & & 399 & & $\$ 16$ & 7.8 & 97 & & 464 & 8.9 & 74 & 9.0 & 91 & & 294 \\
\hline KS & & 78 & & 47 & 0.1 & 73 & & 152 & & 153 & 0.1 & 70 & & 000 & & 00 & 0.0 & 00 & & 132 \\
\hline
\end{tabular}

$=23-1,127-129 \mathrm{~cm}$; plagioclase $5.2=23-1,127-129 \mathrm{~cm}$; plagioclase $6.3=21-1,47-49 \mathrm{~cm} ;$ plagioclase $5.4=21-1,47-49 \mathrm{~cm} ;$ plagioclase $6.5=21-1,47-49 \mathrm{~cm} ;$ plagioclase $7.6=21-1,47-49 \mathrm{~cm} ;$ plagioclase $8.7=15-2,20 \mathrm{~cm}$; plagioclase $1.8=20-2,140-145 \mathrm{~cm}$; plagioclase $1.9=20-2,140-145 \mathrm{~cm}$; plagioclase $2.10=24-3,112-114 \mathrm{~cm} ;$ plagioclase 6 . 
TABLE 4

Microprobe Analyses of Spinels

\begin{tabular}{|c|c|c|c|c|c|c|c|c|c|c|c|c|}
\hline & \multicolumn{2}{|c|}{1} & \multicolumn{2}{|c|}{2} & \multicolumn{2}{|c|}{3} & \multicolumn{2}{|c|}{4} & \multicolumn{2}{|c|}{5} & \multicolumn{2}{|c|}{6} \\
\hline $\mathrm{SiO}_{2}$ & \multicolumn{2}{|c|}{0.00} & \multicolumn{2}{|c|}{0.00} & \multicolumn{2}{|c|}{0.00} & \multicolumn{2}{|c|}{0.00} & \multicolumn{2}{|c|}{0.01} & \multicolumn{2}{|c|}{0.02} \\
\hline $\mathrm{TiO}_{2}$ & \multicolumn{2}{|c|}{0.37} & \multicolumn{2}{|c|}{0.40} & \multicolumn{2}{|c|}{0.66} & \multicolumn{2}{|c|}{0.70} & \multicolumn{2}{|c|}{0.63} & \multicolumn{2}{|c|}{0.52} \\
\hline $\mathrm{Al}_{2} \mathrm{O}_{3}$ & \multicolumn{2}{|c|}{24.66} & \multicolumn{2}{|c|}{22.10} & \multicolumn{2}{|c|}{21.58} & \multicolumn{2}{|c|}{21.59} & \multicolumn{2}{|c|}{21.64} & \multicolumn{2}{|c|}{20.65} \\
\hline $\mathrm{Fe}_{2} \mathrm{O}_{3}$ & \multicolumn{2}{|c|}{2.77} & \multicolumn{2}{|c|}{3.93} & 4. & & & 62 & & & & \\
\hline $\mathrm{FeO}$ & 15 & & 18 & & 17. & & 17 & & 19. & & 20 & \\
\hline $\mathrm{Cr}_{2} \mathrm{O}_{3}$ & 43 & & 43 & & 42. & & 43 & & 42. & & 42 & \\
\hline $\mathrm{MnO}$ & & & & & 0. & & & 36 & & & & \\
\hline $\mathrm{MgO}$ & 13 & & 10 & & 11. & & 11 & & 10. & & & \\
\hline $\mathrm{ZnO}$ & & & & & 0 . & & & & & & & \\
\hline $\mathrm{CaO}$ & & & & & 0 . & & & 02 & & & & \\
\hline Sum & 100 & & 99 & & 99. & & 100 & & 99. & & 99 & \\
\hline $\mathrm{Si}$ & 0.000 & * & 0.000 & * & 0.000 & * & 0.000 & * & 0.003 & * & 0.005 & * \\
\hline $\mathrm{Ti}$ & 0.068 & $*$ & 0.075 & $*$ & 0.124 & $*$ & 0.131 & * & 0.100 & * & 0.099 & * \\
\hline $\mathrm{Al}$ & 7.064 & $*$ & 6.522 & * & 6.369 & * & 6.322 & * & 6.409 & * & 6.181 & * \\
\hline $\mathrm{Cr}$ & 8.288 & $*$ & 8.580 & * & 8.486 & * & 8.547 & * & 8.427 & * & 8.618 & * \\
\hline $\mathrm{Fe} 3+$ & 0.507 & 15.926 & 0.741 & 15.918 & 0.891 & 15.871 & 0.864 & 15.864 & 0.955 & 15.894 & 0.986 & 15.890 \\
\hline $\mathrm{Fe}$ & 3.155 & $*$ & 3.913 & $*$ & 3.667 & $*$ & 3.705 & $*$ & 4.048 & $*$ & 4.380 & $*$ \\
\hline $\mathrm{Mn}$ & 0.060 & $*$ & 0.078 & * & 0.076 & $*$ & 0.076 & * & 0.079 & $*$ & 0.088 & * \\
\hline $\mathrm{Mg}$ & 4.852 & * & 4.084 & $*$ & 4.379 & * & 4.348 & * & 3.975 & * & 3.635 & * \\
\hline $\mathrm{Zn}$ & 0.000 & $*$ & 0.000 & * & 0.000 & * & 0.000 & * & 0.000 & $*$ & 0.000 & * \\
\hline $\mathrm{Ca}$ & 0.005 & 8.072 & 0.005 & 8.080 & 0.005 & 8.128 & 0.005 & 8.135 & 0.003 & 8.104 & 0.005 & 8.109 \\
\hline $\mathrm{O}$ & 32.000 & * & 32.000 & * & 32.000 & * & 32.000 & * & 32.000 & * & 32.000 & * \\
\hline CHRO & 52 & & 54 & & 53. & & 54 & 324 & 53. & & 54 & 94 \\
\hline SPIN & 44 & & 41 & & 40. & & 40 & 185 & 40. & & 39 & 58 \\
\hline MAGN & & 95 & & & & 61 & & 491 & & & & 48 \\
\hline USP & & & & & & 83 & & 788 & & & & 67 \\
\hline SPIN & 92 & & 88 & & 86. & & 86 & 405 & 85. & & 85 & \\
\hline MAGN & & 34 & 10 & & 12. & & 11 & & 12. & & 13 & \\
\hline USP & & 63 & & & & 08 & & 371 & & & & 24 \\
\hline CHRO & 93 & & 91 & & 89. & & 89 & 574 & 88. & & 88 & \\
\hline MAGN & & 18 & & & & 81 & & 55 & 10. & & 10. & \\
\hline $\mathrm{F} / \mathrm{M}$ & & 63 & & & & 55 & & 870 & & & & 29 \\
\hline $\mathrm{F} / \mathrm{FM}$ & & & & & 0.4 & & & 465 & & & & 51 \\
\hline
\end{tabular}

Note: $1=26-1,20-22 \mathrm{~cm}$; spinel $1.2=26-1,20-22 \mathrm{~cm}$; spinel $2.3=26-2,5-10 \mathrm{~cm}$; spinel $1.4=26-2,5-10 \mathrm{~cm} ;$ spinel $2.5=22-2$, $61-63 \mathrm{~cm}$; spinel $6.6=22-2,6163 \mathrm{~cm}$; spinel 7 . 
TABLE 5

Summary of Petrography

\begin{tabular}{|c|c|c|c|c|c|c|}
\hline $\begin{array}{c}\text { Sample } \\
\text { (Interval in } \mathrm{cm} \text { ) }\end{array}$ & Rock Type & Modal Analysis & & Grain Size (mm) & Texture & Alteration \\
\hline $15-2,20$ & Basalt & $\begin{array}{l}\text { Plagioclase } \\
\text { Clinopyroxene } \\
\text { Olivine }\end{array}$ & $\begin{array}{r}50 \\
45 \\
5\end{array}$ & $\begin{array}{l}\text { Phenocrysts }<3 \\
\text { Groundmass }<1\end{array}$ & $\begin{array}{l}\text { Porphyritic } \\
\text { (phenocrysts of } \\
\text { Plag, Cpx, Oliv) }\end{array}$ & $\begin{array}{l}\text { Minor chlorite } \\
\text { in vesicles }\end{array}$ \\
\hline $20-2,140-145$ & Basalt & $\begin{array}{l}\text { Plagioclase } \\
\text { Clinopyroxene } \\
\text { Quench crystals } \\
\text { Olivine }\end{array}$ & $\begin{array}{r}5 \\
5 \\
90 \\
\operatorname{Tr}\end{array}$ & $<1$ & $\begin{array}{l}\text { Microporphyritic } \\
\text { rock consisting } \\
\text { mainly of quench } \\
\text { crystals in vario- } \\
\text { litic texture }\end{array}$ & Fresh \\
\hline $21-1,47-49$ & $\begin{array}{l}\text { Anorthositic } \\
\text { gabbro }\end{array}$ & $\begin{array}{l}\text { Plagioclase } \\
\text { Clinopyroxene }\end{array}$ & $\begin{array}{l}60 \\
40\end{array}$ & $1-6$ & $\begin{array}{l}\text { Plagioclase-Cpx } \\
\text { adcumulate; ex- } \\
\text { solution in Cpx }\end{array}$ & Minor sericite \\
\hline $22-1,17-23$ & Gabbro & $\begin{array}{l}\text { Plagioclase } \\
\text { Pyroxene }\end{array}$ & $\begin{array}{l}60 \\
40\end{array}$ & Phenocrysts $<3$ & $\begin{array}{l}\text { Cumulus plagio- } \\
\text { clase; intercumulus } \\
\text { pyroxene }\end{array}$ & $\begin{array}{l}\text { Partially amphi- } \\
\text { bolitized }\end{array}$ \\
\hline $22-2,61-63$ & Peridotite & $\begin{array}{l}\text { Olivine } \\
\text { Clinopyroxene } \\
\text { Rodingite }\end{array}$ & $\begin{array}{l}70 \\
15 \\
15\end{array}$ & $\begin{array}{l}\text { Olivine } 10-20 \\
\text { Cpx } 1-4 \mathrm{~mm}\end{array}$ & $\begin{array}{l}\text { Cumulus olivine; } \\
\text { intercumulus } \mathrm{Cpx} \\
\text { with minor ex- } \\
\text { solution }\end{array}$ & $\begin{array}{l}\text { Olivine 95\% } \\
\text { serpentinized }\end{array}$ \\
\hline $22-2,80-82$ & Perdotite & $\begin{array}{l}\text { Olivine } \\
\text { Clinopyroxene } \\
\text { Orthopyroxene } \\
\text { Chromite }\end{array}$ & $\begin{array}{l}60 \\
18 \\
22 \\
\operatorname{Tr}\end{array}$ & $\begin{array}{l}\text { Olivine 1-2 } \\
\text { Cpx 8-15 }\end{array}$ & $\begin{array}{l}\text { Cpx exsolved; } \\
\text { olivine cumulus } \\
\text { pyroxenes inter- } \\
\text { cumulus; mesh }\end{array}$ & $\begin{array}{l}\text { Olivine and ortho- } \\
\text { pyroxene partly } \\
\text { serpentinized }\end{array}$ \\
\hline $23-1,46-54$ & Peridotite & $\begin{array}{l}\text { Olivine } \\
\text { Iddingsite } \\
\text { Opx } \\
\text { Cpx } \\
\text { Plag }\end{array}$ & $\begin{array}{l}60 \\
40\end{array}$ & Olivine $10-12$ & $\begin{array}{l}\text { Olivines corroded; } \\
\text { exsolution lamellae } \\
\text { in fresh } \mathrm{Cpx}\end{array}$ & $\begin{array}{l}\text { Olivine totally } \\
\text { serpentinized, } \\
\text { Opx-Cpx-Plag } \\
\text { serpentinized } \\
\text { and rodingitized }\end{array}$ \\
\hline $23-1,91-93$ & Gabbro & $\begin{array}{l}\text { Plagioclase } \\
\text { Clinopyroxene }\end{array}$ & $\begin{array}{l}50 \\
50\end{array}$ & $<2$ & $\begin{array}{l}\text { Ophitic; } \\
\text { minor cataclasis; } \\
\text { plagioclase cumu= } \\
\text { lus; clinopyroxene } \\
\text { intercumulus }\end{array}$ & Fresh \\
\hline $23-1,127-129$ & $\begin{array}{l}\text { Noritic } \\
\text { gabbro }\end{array}$ & $\begin{array}{l}\text { Plagioclase } \\
\text { Pyroxenes }\end{array}$ & $\begin{array}{l}40 \\
60\end{array}$ & $3-15$ & $\begin{array}{l}\text { Adcumulate; } \\
\text { augite and inverted } \\
\text { pigeonite both } \\
\text { with exsolution } \\
\text { lamellae; cata- } \\
\text { clastized }\end{array}$ & Fresh \\
\hline $23-2 ; 78-82$ & Peridotite & $\begin{array}{l}\text { Olivine } \\
\text { Orthopyroxene } \\
\text { Plagioclase } \\
\text { Chromite }\end{array}$ & $\begin{array}{l}70 \\
15 \\
15 \\
\operatorname{Tr}\end{array}$ & $<2$ & $\begin{array}{l}\text { Trace of exso- } \\
\text { lution in altered } \\
\text { Opx }\end{array}$ & $\begin{array}{l}\text { Olivine and ortho- } \\
\text { pyroxene totally } \\
\text { serpentinized; } \\
\text { plagioclase } \\
\text { rodingitized }\end{array}$ \\
\hline $24-2,105-107$ & $\begin{array}{l}\text { Olivine } \\
\text { gabbro }\end{array}$ & $\begin{array}{l}\text { Olivine } \\
\text { Plagioclase } \\
\text { Clinopyroxene }\end{array}$ & $\begin{array}{l}15 \\
50 \\
35\end{array}$ & Generally $<4$ & $\begin{array}{l}\text { Plag poikilitic } \\
\text { in } \mathrm{Cpx}\end{array}$ & $\begin{array}{l}\text { Olivine partly alter- } \\
\text { ed to serpentine } \\
\text { and iddingsite }\end{array}$ \\
\hline $24-3,112-114$ & $\begin{array}{l}\text { Olivine } \\
\text { leucogabbro }\end{array}$ & $\begin{array}{l}\text { Olivine } \\
\text { Plagioclase } \\
\text { Pyroxenes }\end{array}$ & $\begin{array}{r}5 \\
55 \\
40\end{array}$ & $1-6$ & Adcumulate & $\begin{array}{l}\text { Olivine partly } \\
\text { serpentinized }\end{array}$ \\
\hline $24-4,100-106$ & $\begin{array}{l}\text { Olivine } \\
\text { gabbro }\end{array}$ & $\begin{array}{l}\text { Olivine } \\
\text { Plagioclase } \\
\text { Pyroxenes }\end{array}$ & $\begin{array}{l}35 \\
20 \\
45\end{array}$ & $\begin{array}{l}\text { Olivine }<10 \\
\text { Plag }<2 \\
\text { Pyroxene } 4\end{array}$ & $\begin{array}{l}\text { Opx and Cpx } \\
\text { zoned, exsolved } \\
\text { and with kink } \\
\text { bands; olivine and } \\
\text { plagioclase cumulus }\end{array}$ & $\begin{array}{l}\text { Olivine partly } \\
\text { serpentinized }\end{array}$ \\
\hline $26-1,20-22$ & Peridotite & $\begin{array}{l}\text { Olivine } \\
\text { Pyroxenes } \\
\text { Rodingite } \\
\text { Spinels }\end{array}$ & $\begin{array}{r}60 \\
30 \\
5 \\
5\end{array}$ & $3-10$ & $\begin{array}{l}\text { Cumulus olivine } \\
\text { and spinel; inter- } \\
\text { cumulus plagio- } \\
\text { clase mesh; bastite; } \\
\text { pyroxenes with } \\
\text { exsolution }\end{array}$ & $\begin{array}{l}\text { Olivine altered to } \\
\text { serpentine }\end{array}$ \\
\hline
\end{tabular}


D. B. CLARKE, H. LOUBAT

TABLE 5 - Continued

\begin{tabular}{|c|c|c|c|c|c|c|}
\hline $\begin{array}{c}\text { Sample } \\
\text { (Interval in } \mathrm{cm} \text { ) }\end{array}$ & Rock Type & Modal Analysis & & Grain Size (mm) & Texture & Alteration \\
\hline $26-1,70-75$ & Peridotite & $\begin{array}{l}\text { Olivine } \\
\text { Clinopyroxene } \\
\text { Orthopyroxene } \\
\text { Chromite }\end{array}$ & $\begin{array}{l}65 \\
22 \\
13 \\
\mathrm{Tr}\end{array}$ & Generally $<10$ & $\begin{array}{l}\text { Cpx with exsolu- } \\
\text { tion lamellae; } \\
\text { Olivine cumulus; } \\
\text { Cpx intercumulus }\end{array}$ & $\begin{array}{l}\text { Olivine totally } \\
\text { serpentinized; } \\
\text { Opx partly } \\
\text { serpentinized }\end{array}$ \\
\hline $26-2,5-10$ & Peridotite & $\begin{array}{l}\text { Olivine } \\
\text { Pyroxenes } \\
\text { Rodingite } \\
\text { Spinel }\end{array}$ & $\begin{array}{r}80 \\
15 \\
5\end{array}$ & $2-3$ & $\begin{array}{l}\text { Cumulus olivine } \\
\text { and spinel; } \\
\text { intercumulus } \\
\text { pyroxenes and } \\
\text { plagioclase; mesh; } \\
\text { bastite }\end{array}$ & $\begin{array}{l}\text { Olivine totally } \\
\text { serpentinized; } \\
\text { Opx partly } \\
\text { serpentinized }\end{array}$ \\
\hline $26-2,56-71$ & $\begin{array}{l}\text { Olivine } \\
\text { gabbro }\end{array}$ & $\begin{array}{l}\text { Olivine } \\
\text { Plagioclase } \\
\text { Pyroxenes }\end{array}$ & $\begin{array}{l}20 \\
40 \\
40\end{array}$ & $<4$ & $\begin{array}{l}\text { Exsolution lamel- } \\
\text { lae in pyroxenes; } \\
\text { cumulus plagioclase } \\
\text { (and olivine?) }\end{array}$ & $\begin{array}{l}\text { Olivine partly } \\
\text { serpentinized }\end{array}$ \\
\hline $26-2,59-64$ & $\begin{array}{l}\text { Olivine } \\
\text { gabbro } \\
\text { (Pyroxenite) }\end{array}$ & $\begin{array}{l}\text { Olivine } \\
\text { Plagioclase } \\
\text { Pyroxenes }\end{array}$ & $\begin{array}{l}10 \\
20 \\
70\end{array}$ & $<3$ & $\begin{array}{l}\text { Plagioclase cumu- } \\
\text { lus; pyroxenes with } \\
\text { exsolution } \\
\text { lamellae }\end{array}$ & $\begin{array}{l}\text { Olivine } \\
\text { serpentinized }\end{array}$ \\
\hline
\end{tabular}


TABLE 6

Summary of Mineral Chemistry for Intrusive Rocks at Site 334

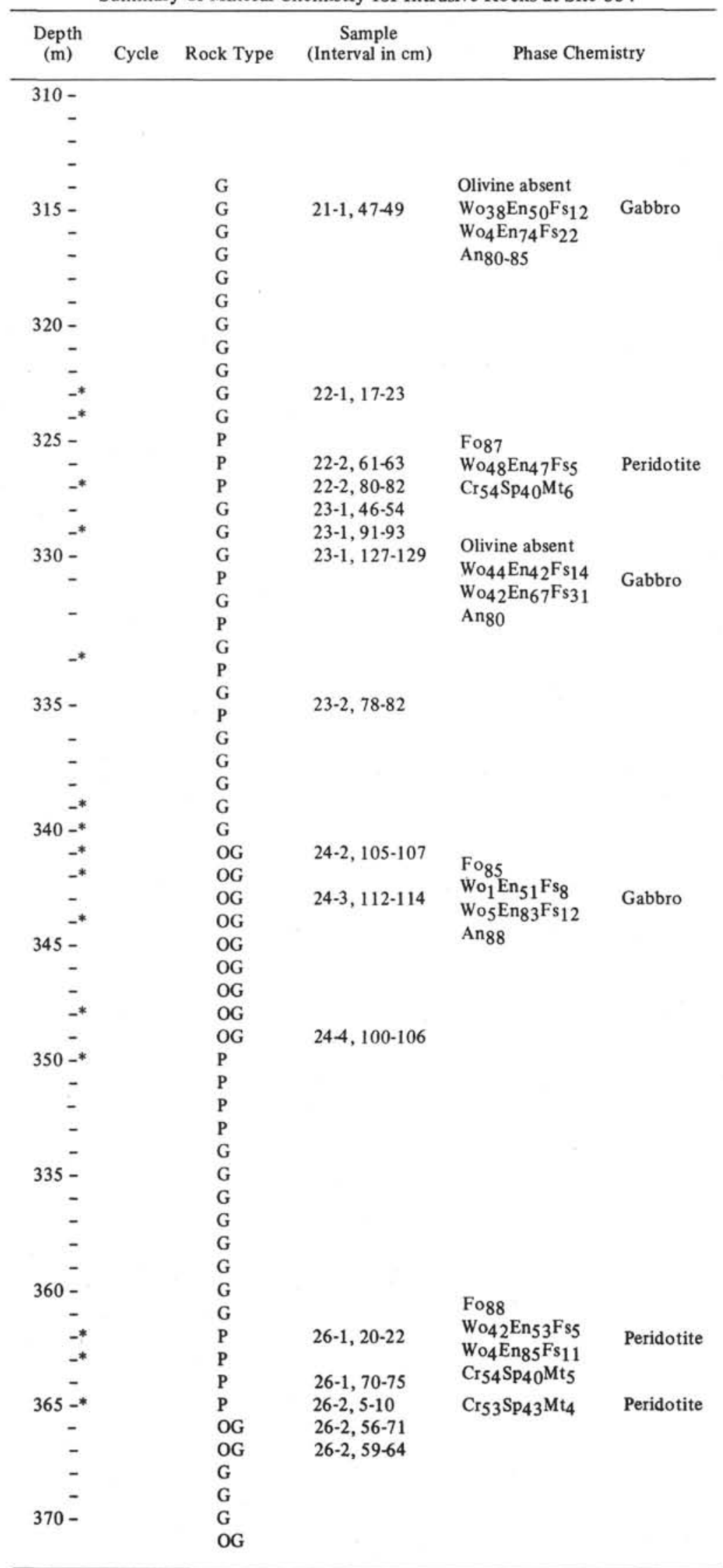

Note: $\mathrm{P}=$ Peridotite $\mathrm{OG}=$ Olivine Gabbro $; \mathrm{G}=\mathrm{Gabbro} ;{ }^{*}=$ Breccia . 